\title{
Dissociation of the blocking of conditioned eyeblink and conditioned fear following a shift in US locus
}

\author{
STEPHANIE L. BETTS, SUSAN E. BRANDON, and ALLAN R. WAGNER \\ Yale University, New Haven, Connecticut
}

\begin{abstract}
Two experiments with rabbits investigated the concordance of two measures of conditioning, eyeblink and potentiated startle, during a blocking sequence with paraorbital shock reinforcement. In both, a shift in the locus of shock from one eye to the other between the conditioning of an element and a compound of that element and a new cue had differential effects on the two measures of conditioning to the new cue. When the shock was unchanged, diminished conditioning in relation to controls (i.e., blocking) was observed on both measures. When the shock was changed, little conditioning was observed in startle, but control-equivalent amounts were observed in eyeblink (i.e., blocking occurred on the former but not the latter). The results are interpreted as showing a dissociation of the associative learning involving the emotive features of Pavlovian reinforcers and that involving the remaining sensory-perceptual features, and more compatible with a diminished US-processing, than with a CSprocessing, view of blocking.
\end{abstract}

"Blocking" is said to occur in Pavlovian conditioning when the reinforced pretraining of one conditioned stimulus (CS) diminishes - that is, blocks - the subsequent, apparent conditioning of another CS when the two are reinforced in compound (Kamin, 1968). Prevailing theoretical interpretations of the phenomenon lead to very different predictions about how the degree of blocking should be affected if some qualitative feature of the reinforcing unconditioned stimulus (US) is modified between the pretraining and the compound phases. The experiments to be reported here were focused on a possibility suggested by a theory of Pavlovian conditioning that we have otherwise proposed to be useful (AESOP; Wagner \& Brandon, 1989): that a shift in the quality of the US will lead to different effects on observed blocking, depending on the type of conditioned response that is measured. Specifically, it was anticipated that a shift in the quality of the US would have different effects on the blocking observed in conditioned emotional responses (CERs), as contrasted with effector-specific conditioned reflexes (CRs).

The blocking paradigm was initially proposed by Lashley (1942) as a way to evaluate whether encouraging the subject to attend to one discriminative stimulus would lead it to fail to attend to, and, hence, fail to learn about, subsequent redundant cues. A prominent group of theories (e.g., Mackintosh, 1975; Pearce \& Hall, 1980; Sutherland \& Mackintosh, 1971) continues to view the phenomenon of blocking in these attentional terms. In line with this think-

This research was supported in part by National Science Foundation Grant BNS-9121094 to A.R.W. Correspondence concerning this article should be addressed to A. R. Wagner, Department of Psychology, Yale University, P. O. Box 208205, New Haven, CT 06520-8205 (e-mail: wagner@yalevm.cis.yale.edu). ing, one might assume that a shift in the character of the US between the pretraining and the compound phase would influence whether an attentional bias would be maintained on compound trials after the shift, with some changes being more likely to cause a change in attention than others. But the reasonable implication for the present concern is that the consequences of any given shift should be reflected similarly on any of the conditioned responses that might be measured, appropriate to the US employed during the compound training. To the degree that the blocked CS remains unattended to, conditioned responding should have no chance to develop. To the degree that the blocked CS is allowed to be attended to, conditioned responding should have as much opportunity to develop as in a comparison treatment without pretraining.

The major alternative to an attentional account of blocking was inspired by Kamin's (1968) investigations of the phenomenon and his suggestion that it might be due to a deficit in processing of the US, rather than the blocked CS, during the compound phase. Wagner $(1969,1976,1978)$ proposed that a stimulus that has its representation active in the memory system at the time of receipt is accorded less processing than it otherwise would receive, and that blocking reflects the diminished "rehearsal" occasioned by a US when it is made to be "expected" by the pretrained CS. This notion is consistent with the manner in which the quantitative learning rule of Rescorla and Wagner (1972; Wagner \& Rescorla, 1972) deduces blocking. By such accounts, a shift in the quality of the US between the pretraining and the compound phase should, obviously, influence blocking: The greater the shift, the more likely it will leave the US unexpected during the compound phase, and thereby less subject to a processing deficit. Although the locus of the processing deficit is presumed 
to be different from that suggested by the aforementioned attentional theories, such theory, without further theoretical adornments, also predicts that blocking should be similarly observed via different measures of conditioning. To the degree that the blocked US is not processed, conditioned responding should have no chance to develop. To the degree that the blocked US is allowed to be processed, conditioned responding should have as much opportunity to develop as in a comparison treatment without pretraining.

In recent years, theorists from both camps (see, e.g., Dickinson, 1980; Mackintosh, 1983; Pearce, 1987; Wagner \& Brandon, 1989) have come to accept a theoretical supposition emphasized by Konorski (1967) that can lead to differential implications, depending on whether one supposes that blocking reflects a deficit in CS processing or in US processing. Konorski proposed that a US should be conceived as represented by multiple nodes in the memory system, with an important distinction being between the representation of the emotional characteristics of the US and the remaining sensory/perceptual attributes of the US. Association of the former with a CS was presumed to be responsible for CERs (which Konorski called "preparatory" responses), whereas association of the latter with a CS was presumed to be responsible for specific CRs (which Konorski called "consummatory" responses).

If the emotional and the sensory/perceptual attributes of a stimulus are separately represented, there is opportunity for a shift in the character of the US to involve a change in one but not the other. And, if the separate representations of the emotional and sensory/perceptual attributes of a US are independently associated with temporally correlated CSs, there is the possibility within a blocking paradigm that a shift in US will lead to diminished blocking on a measure that reflects one but not on a measure that reflects the other. This would be predicted by the US-centered view of blocking, which AESOP (Wagner \& Brandon, 1989) involves. Thus, for example, if pretraining were done with one aversive US and compound training with a qualitatively different, but equally aversive, US, it would be expected that blocking would not be observed on a CR measure specific to the quality of the US, but would be observed, undiminished, on a measure of CER.

Separate studies employing one or the other class of response measures can be viewed as consistent with the preceding prediction. Bakal, Johnson, and Rescorla (1974) pretrained an elemental CS with a footshock US and then shifted for compound conditioning to a qualitatively different, but also aversive, intense noise US. They reported substantial blocking, indicated by a common CER measure involving suppression of appetitive behavior. Stickney and Donahoe (1983), analogously, pretrained an elemental CS with aversive electric shock to the area of one eye and then shifted for compound conditioning to the same shock applied to the area of the other eye. But they employed a conditioned eyeblink measure, and, unlike Bakal et al. (1974), they found no evidence of blocking.

The available studies, however, can be interpreted otherwise. Mackintosh (1983) pointed out that the different training conditions in the separate studies may have made the subjects more or less sensitive to the qualitative changes made to the US. In the then known studies of the CER, subjects were conditioned at long interstimulus intervals that do not appear to support associations between the CS and the sensory/perceptual representations of the USs. Thus, one might argue that blocking occurred in spite of a change in the quality of the US because, under these conditions, there was no disconfirmation of any expectation. In contrast, in the then known studies employing discrete CRs, the subjects were conditioned at short interstimulus intervals presumed to be appropriate for the association of the CS and the sensory/perceptual representations of the US. In this instance, the subjects would be assumed to have had their expectations disconfirmed with a change in the US and, therefore, not to show blocking. Overall, one might read the variation in the results of the several studies as due to variation in whether the US change was surprising. When it was, it could presumably produce "unblocking," either directly, à la Rescorla and Wagner (1972), or by disrupting the attentional bias, à la Mackintosh (1975).

Ganesan and Pearce (1988) also reported blocking using an approach measure, which they presumed to reflect a positive CER, when element conditioning was with either food or water as the US and compound conditioning was with the alternate, similarly attractive US. They argued on the basis of these and other available data involving the CER that blocking will occur with a change in the US, as long as it does not involve a change in the affective properties of the US. To explain the aforementioned findings of Stickney and Donahoe (1983), who observed unblocking when the US was shifted from one eye to the other, they suggested that the shift may have involved an increase in the subjective intensity of the USthe argument being that the animals may have habituated to the US in its initial location and found the same shock in the alternate location transiently more aversive. A variant on this argument might suppose an increase in objective US intensity due to the development of electrode resistance at the pretraining site as compared with the compound training site.

It is possible to evaluate these different interpretations through a conceptual replication of the Stickney and Donahoe (1983) experiment with the inclusion of not only a conditioned eyeblink measure, but a CER measure on the same subjects as well. From the position espoused by Mackintosh (1983), it would be predicted that if unblocking was seen on the eyeblink measure, as it was by Stickney and Donahoe, it would also be seen on the CER measure. From the position espoused by Ganesan and Pearce (1988), a similar concurrence would be expected in the two measures, with the further implication that if steps were taken to preclude differential adaptation to the shock, 
unblocking might not be seen on either measure. In contrast, according to the multiple-node, US-processing account of blocking suggested by AESOP (Wagner \& Brandon, 1989), it would be predicted that unblocking should be seen on the eyeblink measure but not on the CER measure. The two experiments that are reported below present appropriate tests.

\section{EXPERIMENT 1}

The aim of Experiment 1 was to attempt to replicate the differential blocking effects reported by Stickney and Donahoe (1983), with the inclusion of a measure of the CER as well as conditioned eyeblink. However, it was deemed prudent to employ experimental comparisons from which it is safer to conclude that blocking has occurred than the specific comparisons employed by Stickney and Donahoe.

Stickney and Donahoe (1983) compared two groups that received elemental conditioning prior to compound conditioning with a "control" group that received no pretraining prior to the compound phase. They found that a pretrained group given elemental conditioning reinforced by a US to the same eye as that reinforced during the compound phase showed less conditioned responding to the $\mathrm{CS}$ added during the compound phase than did the control group, whereas a pretrained group given elemental conditioning reinforced by a US to the alternate eye as that reinforced during the compound phase did not show such a relative decrement. Although the pattern of data may have resulted from the differential associative learning that was developed to the elemental CS in the three groups prior to compound training, it is also possible that it reflected other differences. Most obvious is the possibility that the pattern reflected differential habituation to the shock US: The unshifted experimental group had experience with the shock employed during the compound phase, whereas the control group had no experience with any shock and the shifted experimental group had only experience with shock to the alternate eye.

Experiment 1 used a variation upon the blocking design previously employed by Wagner and Saavadra (cited in Wagner, 1976) to better equate subjects' experience with shock in the comparison groups. All subjects were given Phase 1, element training with two CSs, A and B, so that $A$ was highly trained and $B$ weakly trained. In Phase 2, four groups were given reinforced training with a target $\mathrm{CS}, \mathrm{X}$, in simultaneous compound with one of the two cues of Phase 1 . For two experimental groups, $X$ was presented in compound with A, the CS with a large amount of Phase 1 training; for two control groups, $X$ was presented in compound with $\mathrm{B}$, the $\mathrm{CS}$ with a small amount of Phase 1 training. For one of the experimental and control groups (designated standard), the compound was reinforced with a US applied to the same location as was the element in Phase 1. For the other experimental and control groups (designated switched), the compound was reinforced with a US applied to the alternate location.
This design allowed the comparison of the behavior of each experimental group, standard or shifted, with that of an appropriate control group that had the same experience with shock and the same overall history of conditioning, but less pretraining of the element that was continued in compound. The question was whether blocking would be similarly observed, or not, in the conditioned eyeblink and potentiated startle.

\section{Method}

Subjects. The subjects were 24 experimentally naive New Zealand white rabbits weighing between 2 and $3 \mathrm{~kg}$ at the start of training. Each rabbit was individually housed and maintained with ad-lib food and water, except during experimental sessions. The experiment was run in three replications, with 2 animals from each of the four treatment groups in each replication. One animal from Group Control Switched was eliminated from the study because of illness after two sessions of Phase 1 training, and its data were excluded from all data description and analyses.

Apparatus. Training and testing were conducted in eight identical $66 \times 48 \times 48 \mathrm{~cm}$ isolation chambers, allowing 8 animals to be run concurrently. Each chamber was completely lined with aluminum foil and illuminated by a $1-W$ neon bulb to provide a homogeneous visual surround. Ventilation fans provided a constant background masking noise that raised the ambient sound pressure level to approximately $70 \mathrm{~dB}$ (re $20 \mu \mathrm{N} / \mathrm{m}^{2}$ ). During experimental sessions, the rabbit was loosely restrained within the chamber in a 51 $\times 18 \times 14 \mathrm{~cm}$ Plexiglas box, from which its head protruded.

There were three CSs, each 1,050 msec in duration. A visual CS was provided by an interrupted light ( 8 flashes/sec), generated by a strobe lamp located behind the subject so as to reflect diffusely from the walls of the chamber. An auditory CS was provided by a $3000-\mathrm{Hz}$ tone that raised the sound pressure level by $20 \mathrm{~dB}$, delivered through a $9-\mathrm{cm}$ speaker located behind the subject on the rear wall of the chamber. A vibratory CS was provided by a $60-\mathrm{Hz}$ oscillation of a hand massager mounted on the floor of the restraint box so as to maintain firm contact with the subject's chest.

The training US and the startle probe both were $50 \mathrm{msec}$ in duration and, when scheduled in the presence of the $C S$, delivered $1,000 \mathrm{msec}$ after CS onset. The shock US was a $100 / \mathrm{sec}$ train of 4-mA square-wave pulses produced by a constant-current generator and delivered to either the left or right paraorbital region through no. 32 gauge, stainless steel sutures that were implanted in the skin about the orbit of the designated eye. One suture was located approximately $5 \mathrm{~mm}$ ventral to the extreme nasal extent and the other approximately $5 \mathrm{~mm}$ caudal to the extreme lateral extent of the eye. The startle-producing stimulus was a roughly square-waved pulse of air, measuring $5.51 \times 10^{4} \mathrm{~N} / \mathrm{m}^{2}$, delivered from a $1.0-\mathrm{mm}$ (I.D.) polyethylene tubing that was secured to the inner surface of the rabbit's pinna and directed to the auditory canal (see below). The air delivery was controlled by a solenoid valve and calibrated in duration and peak intensity via a Statham pressure transducer placed at the orifice of the delivery tubing proximal to the subject. All stimuli were under the control of Tektronix 160 -series pulse generators and associated computer equipment.

Closure of one of the subject's eyes was monitored via an adaptation of the photoresistive transducer described by Gormezano and Gibbs (1988). The transducer, taped directly to the animal's head, communicated with the lid by a length of thread tied to a small permanent recording suture loop. Movements of the eyelid turned a counterweighted wheel affixed to the axle of the transducer. The resulting signal was displayed on a polygraph, adjusted so that a 0.5 $\mathrm{mm}$ eyelid closure produced a $1-\mathrm{mm}$ deflection of a recording pen.

The startle response to the airpuff was measured with an Astatic Model 16 phonocartridge taped to the subject's head. The cartridge held a metal rod, $1 \mathrm{~mm}$ in diameter and $50 \mathrm{~mm}$ in length, in place 
of a phonograph needle. When sudden movements of the subject's head caused the metal rod to oscillate, the cartridge produced a voltage proportional to the vigor of the movement. The voltage output from the movement transducer was graphically recorded on the same polygraph as the eyeblink, with a gain setting of $1 \mathrm{~V} / \mathrm{mm}$.

Preparation. On the day prior to training, the dorsal surface of the rabbits' heads and the regions surrounding their right and left eyes were shaved. US electrodes then were implanted according to each animal's initial training condition, with placement balanced within each group; that is, for half the subjects in each group, electrodes were attached to the right paraorbital region, and for the remaining subjects, they were attached to the left paraorbital region. Right and/or left eyelid sutures were implanted at this time to match the eventual recording requirements in each group. After Session 6, all subjects received new electrodes to be used during compound conditioning: the animals in the two switched groups were implanted with electrodes on the side opposite to the original side; the animals in the two standard groups had their electrodes replaced on the same side, at sites $2 \mathrm{~mm}$ from the location of the original electrodes.

Prior to startle testing, the inner surface of the rabbit's pinna was cleaned with ethanol to facilitate attachment of the air delivery tube, and the auditory canal was cleared of wax and any debris. The tube was secured with vinyl tape from the tip of the pinna, along the central artery, to end approximately $1 \mathrm{~cm}$ from the external auditory meatus. Effectiveness of the placement was checked by ensuring that the rabbit would shake its head in response to a continuous stream of air delivered from a syringe through the attached tubing. 1

Training. Training was conducted in two phases. In the first phase, all subjects were given $448 \mathrm{CS}$-US pairings with one CS, designated $\mathrm{A}$, and 12 similar pairings with another $\mathrm{CS}$, designated $B$. The first training session contained a total of 33 pairings, 32 with $A$ and 1 with $B$, where $B$ occurred after 10 A trials. There followed five daily sessions, each with $80 \mathrm{~A}$ trials and $2 \mathrm{~B}$ trials, where the position of the $B$ trials was varied. Phase 1 training ended with 16 A trials and $1 \mathrm{~B}$ trial in the first part of Session 7. For half the subjects in each group, the light CS was assigned as $\mathrm{A}$, and the vibratory $\mathrm{CS}$ was assigned as $\mathrm{B}$, whereas for the remaining subjects, this was reversed.

Phase 2 training began immediately after the completion of Phase 1 training in Session 7. It involved 80 reinforced compound trials in that session, 80 reinforced compound trials in the next session, and, finally, 16 reinforced compound trials in the last session prior to testing. The compound consisted of the tone $\mathrm{CS}, \mathrm{X}$, presented simultaneously with one of the pretrained elements, A or B. Subjects in Groups Control Standard and Control Switched were given BX compound training, whereas subjects in Groups Experimental Standard and Experimental Switched were given AX compound training. For the subjects of Groups Control Standard and Experimental Standard, the locus of US application in this phase was the same as in Phase 1 training. For the subjects of Groups Control Switched and Experimental Switched, the locus of US application was to the opposite paraorbital region from that in Phase 1 . The intertrial intervals during Phase 1 and Phase 2 were consistently 2 min.

Animals were monitored for CRs on the eye ipsilateral to the location of the US for the element or compound training in effect. An exception was in Session 7 when the locus of US application was changed for some subjects between element and compound training in the midst of the session: To avoid any interruption associated with repositioning the recording gear, all recording was from the eye that was reinforced during the compound trials.

Testing. Testing was initiated after the aforementioned 16 reinforced training trials with AX or BX at the start of Session 10, and after a single adaptation preexposure to the airpuff alone. Subsequent trials included a total of four test presentations of each of the puff alone, $\mathrm{X}$ alone, and $\mathrm{X}$ terminating with the puff. The tests were presented in a different pseudorandom order for each subject in a group, with the common restriction that each block of three test trials included one of each of the test types. To maintain performance during testing, a reinforced $\mathrm{AX}$ or $\mathrm{BX}$ compound trial appropriate to the subject's training was inserted between all adjacent test trials. The intertrial interval was lengthened in this phase to $4 \mathrm{~min}$.

Half the animals in each group received the airpuff to the ear that was on the same side as the shock had been applied in Phase 2, and half received it on the other side. All animals were monitored for eyelid closure on the eye that was reinforced during compound training.

Scoring and data analysis. A conditioned eyelid response to the CS was scored when the graphic record indicated an eyelid closure of $1.0 \mathrm{~mm}$ or more, relative to the pre-CS baseline, during the interval from 40 to $1,000 \mathrm{msec}$ following CS onset. The amplitude of the startle response was defined as the maximum, peak-to-peak voltage recorded within the 300 -msec interval that began with the activation of the airpuff solenoid.

Conditioned eyelid responding is reported as the percentage of trials with a scorable CR to the CS in question. The startle data were rendered as relative frequency scores so that they could be reported similarly. A startle response was scored as "potentiated," or not, on each test trial in which the puff was presented in the presence of $X$, according to whether its amplitude exceeded, or not, the median amplitude of the startle responses observed on the several puffalone test trials for that subject. It should be recognized that by this measure $50 \%$ would indicate equivalent startle to the puff in the presence and absence of $X$, so that only percentages in excess of this would be presumptive of fear conditioning to $X$.

Statistical analyses consisted of analyses of variance and planned contrasts performed as warranted by a priori hypotheses. All tests referred to as significant are associated with $p<.05$ and are twotailed except as noted.

\section{Results and Discussion}

The left panel of Figure 1 presents the mean percentage of the trials in which an eyelid CR, as measured ipsilateral to the location of US application, occurred to A and $B$ in each session of Phase 1 , averaged across the four groups. The right panel of Figure 1 presents the mean percentage frequency of ipsilateral eyelid CRs to $\mathrm{AX}$ or $\mathrm{BX}$ in each session of Phase 2, plotted separately for each of the four groups.

Inspection of the Phase 1 percentages indicates that high levels of responding developed to the more frequently reinforced A cue, as compared with very low levels of responding to the less frequently occurring $B$ cue. This was true for all four groups. Analysis of variance confirmed that the overall superiority of responding to A as compared with $B$ was highly reliable $[F(1,19)=62.25]$, while revealing no significant main effects or interactions involving the different groups $(F \mathrm{~s}<1)$.

Figure 1 does not include the data from the final 17 trials ( 16 with A and 1 with B) of Phase 1 training at the beginning of Session 7, prior to the shift to compound training, because recording was then made from different eyes for the two standard groups versus the two shifted groups. The data are congruent, however, with the lateralized character of the eyeblink $\mathrm{CR}$, being much more apparent when recorded on the eye ipsilateral to the location of the training US than on the alternate eye (see Brandon, Betts, \& Wagner, 1994). Groups Control Standard and Experimental Standard, for which records were taken from the eye ipsilateral to the locus of the Phase 1 US, showed mean percentage CRs to A of 75.8 and 79.9, respectively. In comparison, Groups Control Switched and Experi- 

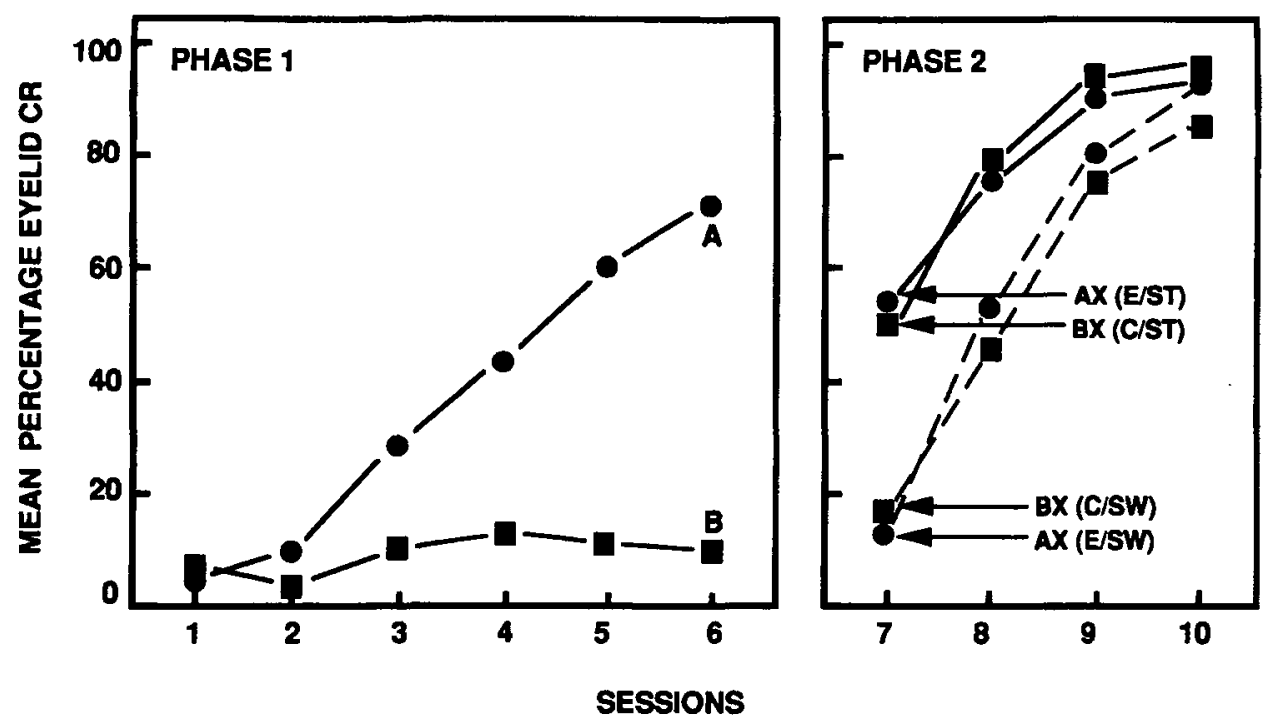

Figure 1. The left panel depicts the mean percentage conditioned eyelid closures to $A$ and $B$ for all subjects in successive training sessions of Phase 1 of Experiment 1 . The right panel depicts the mean percentage conditioned eyelid closures for Groups Experimental Standard (E/ST), Control Standard (C/ST), Control Switched (C/SW), and Experimental Switched (E/SW), in successive training sessions in Phase 2, Experiment 1.

mental Switched, for which records were taken from the eye contralateral to the locus of the Phase 1 US, showed mean percentage CRs of 18.4 and 8.3 , respectively. (No subject in any group responded to cue $B$ in this session.) This difference in the percentage CRs recorded from the different eyes of the two standard versus the two switched groups was highly reliable $[F(1,21)=44.37]$.

During Phase 2 compound training, the levels of eyeblink responding observed on the reinforced eye continued to reflect the training differences between the standard and the switched groups. As shown in Figure 1, the subjects in Groups Control Standard and Experimental Standard, which received BX and AX, respectively, reinforced with a US at the same location as in Phase 1 , showed greater eyeblink percentages than did the subjects in Groups Control Switched and Experimental Switched, which received $B X$ and $A X$, respectively, reinforced with a US at the alternate location relative to Phase 1 . The difference in observed eyeblink responding between the standard and switched groups was statistically significant over Sessions 1-3 [Fs $(1,19)>7.35]$, but not in Session 4 $(F<1)$.

Although there were large within-group differences in responding to $A$ versus $B$ alone at the end of Phase 1 , as indicated above, this did not carry over into any sizable advantage in the responding of the groups conditioned with $\mathrm{AX}$ as compared with $\mathrm{BX}$ during Phase 2 . Difference was not necessarily anticipated between the two switched groups, since responding on the eye reinforced during Phase 2 was negligible to either $\mathbf{A}$ or $\mathbf{B}$ alone at the end of Phase 1 . However, difference was anticipated between the experimental standard group that received $\mathrm{X}$ in compound with $A$, a cue to which it had previously shown a high level of response on the reinforced eye, in comparison with the control standard group that received $\mathrm{X}$ in compound with $B$, a cue to which it had previously shown little response. Wagner and Saavadra (Wagner, 1976) reported a substantial difference in a corresponding comparison, but any like difference in Phase 2 of Experiment 1 was quite fleeting: Over the first block of 10 trials of Phase 2 in Session 7, the experimental standard group responded to $\mathrm{AX}$ on $28 \%$ of the trials, whereas the control standard group responded to BX on only $3 \%$ of the trials [and even this difference was not statistically reliable; $t(10)=1.49$.

The data of principal interest, from the test session, are displayed in Figure 2. The top graph shows, for each of the four groups, the mean percentage of test trials with the $X$ cue alone in which an eyeblink CR was observed. The bottom graph, similarly, shows the mean percentage of test trials when startle was probed in the presence of $\mathrm{X}$ in which startle was greater than the median startle to the probe alone.

It is apparent in the eyeblink data summarized in Figure 2 that there was less conditioned responding by the experimental standard group than by the three remaining groups, which behaved relatively equivalently. That is, a blocking effect was evident in the diminished conditioned eyeblink responding to $X$ alone observed in the experimental standard group as compared with the control standard group, replicating the findings reported by Wagner and Saavadra with this particular experimental comparison. In contrast, there was no similar decrement in the eyeblink performance of the experimental switched group to that in the experimental standard group. The apparent blocking of eyeblink performance observed in the 

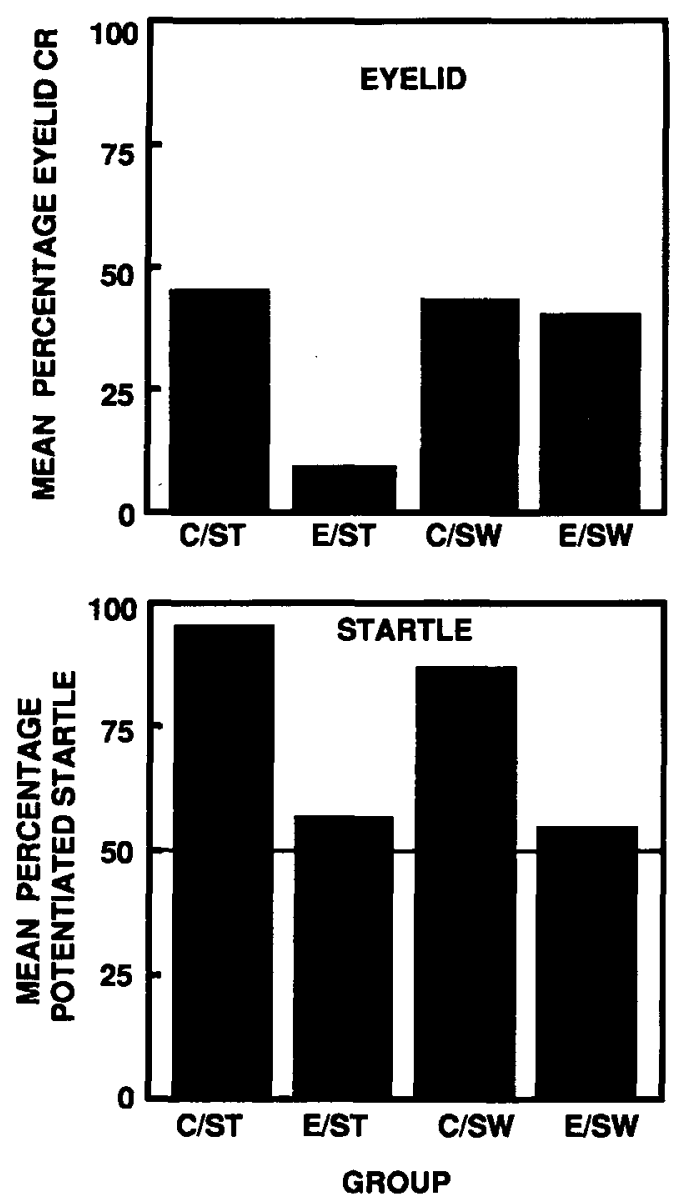

Figure 2. Mean percentages conditioned eyelid closures (top panel) and potentiated-startle responses (bottom panel) for Groups Control Standard (C/ST), Experimental Standard (E/ST), Control Switched (C/SW), and Experimental Switched (E/SW), in the test session of Experiment 1.

experimental standard group, but not in the experimental switched group, when each is compared with the control standard group, replicates the pattern of data reported by Stickney and Donohoe (1983). There was also no apparent blocking in the performance of the experimental switched group in comparison with that of the control switched group. Statistical analyses indicated that CR frequency was reliably less in Group Experimental Standard than in Group Control Standard $[t(10)=2.53]$ but not reliably different in Group Experimental Switched than in Group Control Switched $(t<1)$. The difference between Groups Experimental Standard and Experimental Switched was reliable $[t(10)=2.23]$.

The pattern of data involving the potentiated-startle measure, as summarized in the lower panel of Figure 2, is quite different from that involving the eyeblink CR. As may be seen, in this case, each of the experimental groups showed less potentiated startle than did its respective control group. That is, blocking was apparent when one element of the compound had previously been frequently rather than infrequently reinforced with paraorbital shock, independent of the constancy versus shift in the location of the shock between the pretraining and compound phases. Preliminary analysis indicated no reliable $\left(F_{\mathrm{s}}<1\right)$ or otherwise notable differences among the four groups in either the frequency or the amplitude of the startle response to the airpuff alone (data not shown). However, analysis of the potentiated startle data in the presence of X, depicted in Figure 2, confirmed that the decrement in the experimental standard group in comparison with the control standard group was reliable $[t(10)=$ $2.82]$, as was the decrement in the experimental switched group in comparison with the control switched group $[t(9)=2.52]$. There was no reliable difference in the potentiated-startle performance between the switched and standard experimental groups $(t<1)$.

The results of Experiment 1 simultaneously support the observations of Stickney and Donahoe (1983) and Bakal et al. (1974), which might have been thought to be discordant: The conditioned eyeblink data confirm the conclusions of Stickney and Donahoe that blocking of this measure does not occur when the location of the US is changed between the pretraining and the compound phases, as it does when the location of the US is unchanged; the potentiated-startle data are equally consistent with the findings of Bakal et al., who reported that blocking of the CER occurred in spite of a shift in the quality of the aversive US-that is, blocking of potentiated startle was observed when the US was changed in location between the pretraining and the compound phases, as well as when unchanged.

One could summarize the critical difference in the two measures of Experiment 1 as involving whether blocking was observed in the performance of the experimental shifted group relative to the comparison groups, noting that it was observed in the startle measure but not in the eyeblink CR. From such a summary one could be led to wonder whether the CER might, simply, have been more sensitive than the eyeblink CR in allowing detection of the same underlying effect. We think that this reasoning must be rejected on the basis of the full pattern of group relationships that have been described. In the startle measure, the responding of the experimental switched group was reliably less than that of the control switched group and not reliably different from that of the experimental standard group; in the eyeblink measure, the responding of the experimental switched group was not reliably less than that of the control switched group but was reliably greater than that of the experimental standard group. Thus, if one wished to say that the CER was more sensitive than the conditioned eyeblink in detecting a difference between the experimental switched and control switched groups, one would also have to say that the CER was less sensitive than the eyeblink in detecting a difference between the experimental switched and experimental standard groups. It is more parsimonious to accept the apparent dissociation: Shifting the locus of the US between pretraining and compound conditioning prevented blocking 
otherwise seen of the conditioned eyeblink but not of the CER.

\section{EXPERIMENT 2}

Experiment 2 was conducted to evaluate the reproducibility of the apparent dissociation of blocking seen in the two response measures of Experiment 1. It was also designed to comment more effectively upon the factor that Ganesan and Pearce (1988) have suggested may have been critical to the unblocking with a shift in US locus that was observed in the eyeblink measure by Stickney and Donahoe (1983) and was replicated in Experiment 1. Ganesan and Pearce noted that unblocking may have resulted in the experimental shifted subjects of Stickney and Donahoe because the US in this case had never been experienced and subjected to habituation. Experiment 1 showed that unblocking occurred in the shifted experimental condition when comparison was with a shifted control treatment that had also had no prior experience with the US, and that the unblocking occurred only in the eyeblink measure, not in the CER. Thus, it does not appear possible to account for the pattern of data in the simple fashion proposed by Ganesan and Pearce. Nonetheless, it remains possible that the unblocking that did occur in the eyelid responding of the switched groups was dependent on the fact that the US that was involved in Phase 2 compound conditioning was not habituated as it was in the standard groups.

To address this possibility, all animals in Experiment 2 were trained in Phase 1 with three cues: CS A was reinforced by US application to one paraorbital region; CS B was reinforced by US application to the alternate paraorbital region; CS C was not reinforced. Thus, all animals, prior to Phase 2, had equivalent conditioning experience with the US applied to both paraorbital regions. In Phase 2, the animals were segregated into three groups and given compound conditioning, involving a new $C S, X$, in compound with either $A, B$, or $C$, and the same US. For the subjects of Group Experimental Standard, it was arranged that AX was reinforced by a US on the same side as was $A$ in Phase 1. For the subjects of Group Experimental Switched, it was arranged that BX was reinforced by a US on the opposite side to that of B in Phase 1 - that is, also on the same side as was $\mathrm{A}$ in Phase 1. And, for the subjects of Group Control, CX similarly was reinforced with a US whose locus of application was to the side of A in Phase 1.

From the results of Experiment 1, it was expected that both the experimental standard group and the experimental shifted group would show blocking of the acquisition of potentiated startle in comparison with the control group, since both of the experimental groups, but not the control group, experienced $\mathrm{X}$ in compound with a CS that had been paired with an aversive US during Phase 1. Likewise, from the results of Experiment 1, it was expected that the experimental standard group would show blocking of the acquisition of the conditioned eyeblink response in comparison with the control group, but that the experimental shifted group would not, since the experimental standard group uniquely experienced $\mathrm{X}$ in compound with a CS that had been paired with a US to the same location as obtained during compound conditioning.

\section{Method}

Subjects and Apparatus. The subjects were 18 experimentally naive rabbits, obtained and maintained as those of Experiment 1 . The experiment was run in three replications of 6 rabbits each, 2 from each of the three treatment groups. The apparatus was the same as in Experiment 1, except for providing for three auditory CSs, including a broad-spectrum noise and a series of clicks ( 8 pulses $/ \mathrm{sec}$ ) in addition to the $3000-\mathrm{Hz}$ tone previously employed, and all increasing the ambient sound level approximately $20 \mathrm{~dB}$ as measured at the locus of the animal's head. The visual stimulus employed in Experiment 1 was used as the fourth CS.

Preparation. The animals were habituated and prepared like those of the previous experiment, except that all animals were implanted with left and right eyelid sutures and left and right US electrodes on the day prior to the initiation of training, and were so maintained throughout the experiment.

Training. Except as noted, training used the same CS and US parameters and the same interstimulus and intertrial intervals as in Experiment 1. In Phase 1, all subjects received an equal number of training trials with each of the three auditory stimuli, designated A, $B$, and $C$, in which $A$ was paired with shock to one paraorbital region, $\mathrm{B}$ was paired with shock to the alternate paraorbital region, and $C$ was nonreinforced. The location of the US for CS A was to the right paraorbital region for half the animals, and to the left for the remaining animals. The location of the US for B was opposite that for A, for all subjects. The tone, noise, and click stimuli were equally often designated as A, B, and C.

Within daily sessions, trials with $\mathrm{A}, \mathrm{B}$, and $\mathrm{C}$ were irregularly ordered with the restriction that each trial type occurred once in every block of three trials. Phase 1 training completely occupied Sessions $1-12$, the first containing a total of 30 trials, and the next 11 , each containing a total of 90 trials; Phase 1 training was completed with 15 total trials as the start of Session 13.

Phase 2 training began immediately following the completion of Phase 1 training in Session 13. It involved 45 compound conditioning trials in that session, 60 in Session 14, and, finally, 16 in Session 15 prior to testing. The compound consisted of the light $\mathrm{CS}, \mathrm{X}$, presented simultaneously with one of the pretrained elements-A, B, or C-consistently reinforced. For subjects in the experimental standard group, the AX compound was presented and terminated with a US to the same paraorbital region as had been assigned to $\mathrm{A}$ in Phase 1. For subjects in the experimental switched group, the BX compound was presented and terminated with a US to the opposite paraorbital region as had been assigned to $B$ in Phase 1 , which was the side assigned to $A$ in Phase 1 . For subjects in the control group, the CX compound was presented and terminated with a US that similarly was designated for A during Phase 1.

Testing. Testing was initiated after the aforementioned 16 reinforced compound trials with AX, BX, or CX that began Session 15 and after a single adaptation preexposure to the airpuff alone. Subsequent test trials included a total of four tests, each with the airpuff alone, $\mathrm{X}$ alone, and $\mathrm{X}$ terminating with the puff, as in Experiment 1. The tests were presented in a different pseudorandom order for each subject in a group, with the restriction that each block of trials include one of each of the test types.

Response measures. During the 12 sessions of Phase 1 , records were obtained from either the left or the right eye of any subject in each session. Half of the subjects were recorded in the daily order LRRLLRRLLRRL, and half in the order RLLRRLLRRLLR, so that data were available from the two eyes of every subject in each successive pair of sessions. This allowed analysis of the development of 
eyeblink responses to $A, B$, and $C$ from both the eye to which the US was applied in reinforcement of $A$ and in reinforcement of $B$. In the last 15 trials of Phase 1 , in Phase 2 compound training and in testing, records were obtained on every trial from the eye ipsilateral to the locus of the US employed with the compound stimulus.

\section{Results and Discussion}

The eyelid CRs that were observed during the several phases of Experiment 2 were highly lateralized; that is, they were more apparent on the eye ipsilateral, rather than contralateral, to the locus of US application for each reinforced element or compound, as they were in Experiment 1. This tendency was the most impressive during Phase 1, when the subjects came to respond predominately with closure of one eye to $A$ and of the other eye to $\mathrm{B}$ as a result of the stimuli's being reinforced by USs to the alternate paraorbital regions (see Brandon et al., 1994). During the last two sessions of Phase 1, the mean percentage of CRs to A was 82.1 on the eye reinforced in A; it was 52.6 on the eye otherwise reinforced in $\mathrm{B}$. The similar percentage of CRs to B was 89.0 on the eye reinforced in B; it was 42.3 on the eye otherwise reinforced in A. This discriminated lateralization of the eyeblink $\mathrm{CR}$ to $\mathrm{A}$ and $\mathrm{B}$ was highly reliable $[t(15)=9.39]$.

In contrast to the high levels of (differentiated) eyeblink responding that came to be generated by A and B, little responding occurred to the nonreinforced $C$. The left panel of Figure 3 presents the mean percentage CRs observed on the eye ipsilateral to the location of the respective US for $\mathrm{A}$ and $\mathrm{B}$, and equally from the two eyes for the nonreinforced $C$, over successive 2-day blocks of Phase 1 for all subjects. Statistical analysis of these data confirmed that there was reliably less responding to $\mathrm{C}$ than to either $\mathrm{A}$ or $\mathrm{B}\left[F_{\mathrm{S}}(1,30)>109.0\right]$, and no significant difference between the latter two cues $(F<1)$. There were no notable differences among the three groups that received differential treatment in Phase $2(F \mathrm{~s}<1)$.

The right panel of Figure 3 summarizes the eyeblink performance of each of the three groups during Phase 2 . Plotted are the mean percentage eyeblink responses ipsilateral to the location of the US during this phase, over successive 15-trial blocks. The animals of Group Standard, which received $\mathrm{AX}$ reinforced at the same location as $\mathrm{A}$, responded at a high frequency throughout. The animals of Group Switched, which received BX reinforced at the alternate location from $B$, showed a transient lower level of responding to the compound. The animals of Group Control, which received $\mathrm{CX}$ reinforced following the nonreinforcement of $\mathrm{C}$, responded least in the early trials, but quickly came to the same high levels as did the remaining groups. Statistical analysis of this performance showed a reliable interaction of groups with trial blocks $[F(16,120)=4.27]$. Subsequent comparisons revealed that response frequency varied significantly among the groups in the first block of Phase $2[F(2,15)=13.52]$, with the response of Group Control being reliably less than that of both Group Standard $[t(10)=4.75]$ and Group Switched $[t(10)=2.53]$, which two groups also differed reliably $[t(10)=3.37]$.

The data from the test session are shown in Figure 4. The top graph shows, for each of the three groups, the mean percentage of test trials with the $X$ cue alone in which an eyeblink $C R$ was observed. The bottom graph, similarly, shows the mean percentage of test trials when startle was probed in the presence of $X$ in which startle was greater than the median startle to the probe alone.
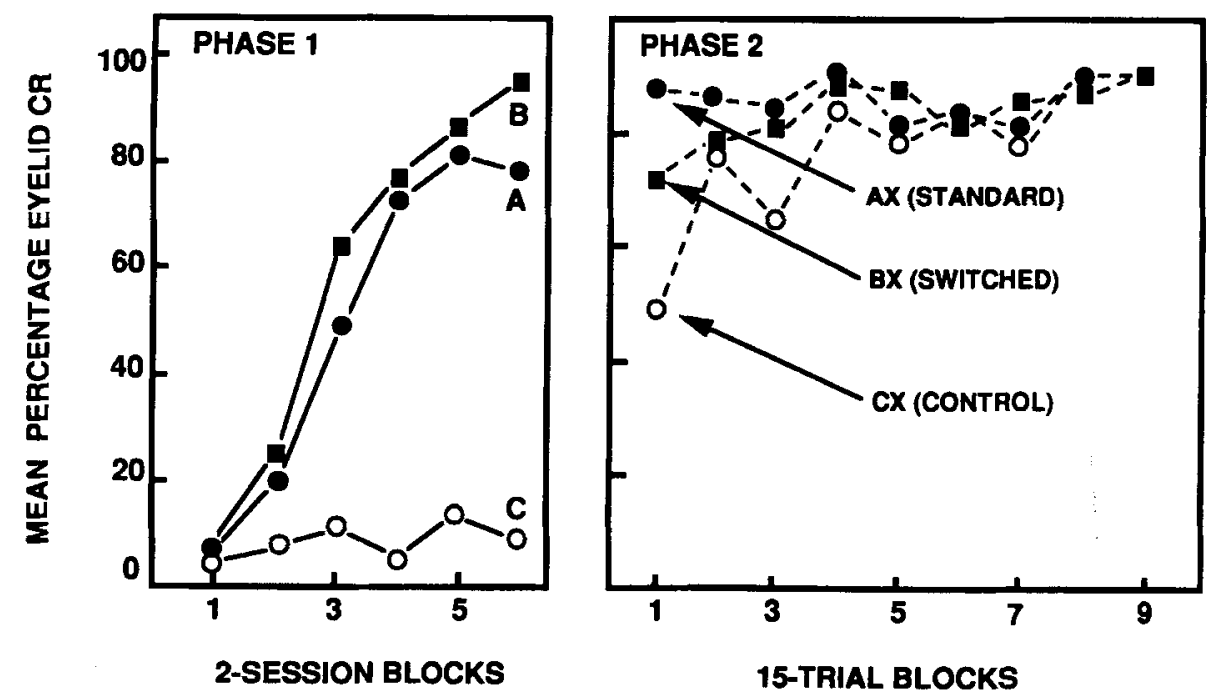

Figure 3. The left panel depicts the mean percentage conditioned eyelid closures to $\mathrm{A}$, $\mathrm{B}$, and $\mathrm{C}$ for all subjects in successive two-session blocks of Phase 1 of Experiment 2 . The right panel depicts the mean percentage conditioned eyelid closures for Group Standard, trained with AX, Group Switched, trained with BX, and Group Control, trained with CX, over successive 15-trial blocks of Phase 2 of Experiment 2. 

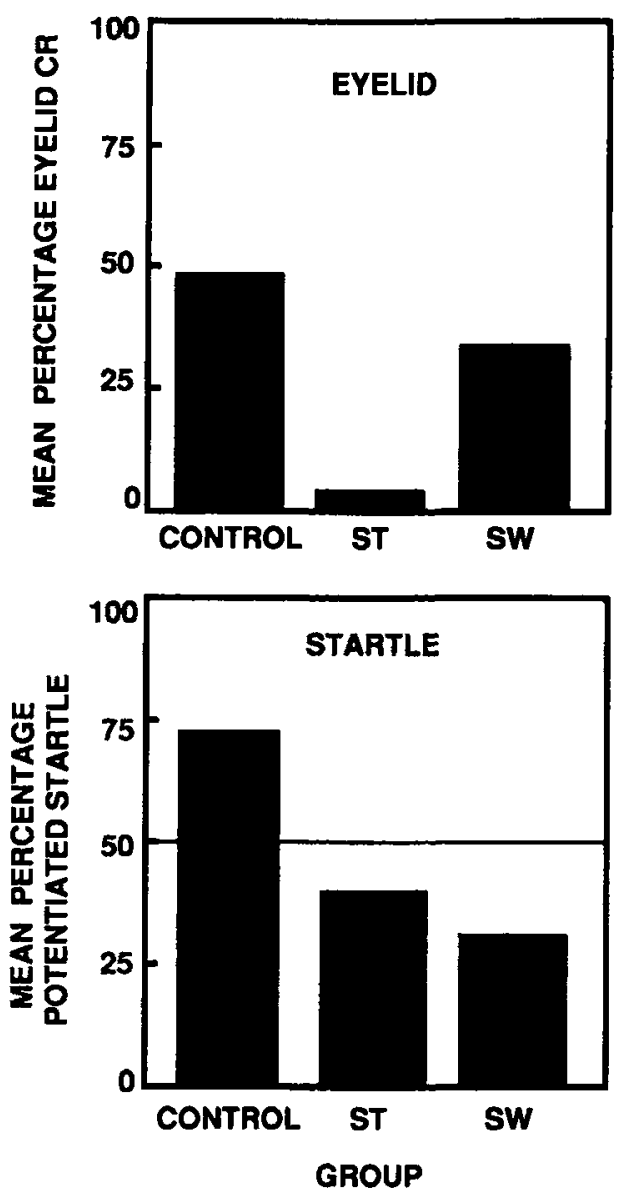

Figure 4. Mean percentages conditioned eyelid closures (top panel) and potentiated startle (bottom panel) for Groups Control, Standard (ST), and Switched (SW), in the test session of Experiment 2.

It can be seen in the eyeblink data summarized in Figure 4 that there was a clear blocking effect in the attenuated responding to $\mathrm{X}$ alone of the standard group in comparison with the control group. In contrast, a blocking effect was less evident in the responding of the switched group, which gave more eyeblink responses than did the standard group and not markedly less than the control group. Planned contrasts confirmed that the CR frequency of Group Standard was reliably less than that of Group Control $[t(10)=3.07]$, whereas that of Group Switched was significantly greater than that of Group Standard $[t(10)=1.87$, one-tailed], and not reliably less than that of Group Control $(t<1)$.

The startle measure summarized in the lower panel of Figure 4 again showed a different pattern than the eyeblink: Apparent blocking was observed in both Group Standard and Group Switched, each evidencing less potentiated startle than did Group Control. Indeed, it may be seen that the observed decrement was somewhat greater in the case of Group Switched than in the case of Group Standard. Preliminary analysis indicated no reliable $(F \mathrm{~s}<1)$ or otherwise notable differences among the three groups in either the frequency or the amplitude of startle response to the airpuff alone (data not shown). Planned comparisons confirmed that the mean frequency of potentiated startle in the presence of X of Group Standard was reliably less than that of Group Control $[t(10)=$ 2.53]. The potentiated startle of Group Switched was also reliably less than that of Group Control $[t(10)=2.58]$, being not significantly different from that of Group Standard $[t(10)=1.38]$.

The test results of Experiment 2 support those of Experiment 1 in indicating that blocking of the conditioned eyeblink response and blocking of the potentiated startle are dissociable. On the eyeblink measure, training $\mathrm{X}$ in compound with a previously reinforced $\mathrm{CS}$, as compared with a previously nonreinforced $\mathrm{CS}$, resulted in blocked acquisition to $\mathrm{X}$ only when the CS was reinforced by a US to the same paraorbital region as that for the compound. On the startle measure, training $X$ in compound with a previously reinforced $C S$, as compared with a previously nonreinforced $\mathrm{CS}$, resulted in blocked acquisition to $\mathrm{X}$ when the CS was reinforced by a US to the same or to the alternate paraorbital region as that for the compound. Conclusions are the easier to draw since the design of Experiment 2 ensured that all subjects had the same overall experience with discriminated conditioning in the pretraining phase, involving USs to both of the paraorbital regions and the same training with one of the USs in the compound phase, differing only in the historical relationship between that US and the CS that was carried over from the pretraining to the compound phase. That is, the differences between the results seen with the two response measures are very likely to represent differences in associative blocking, rather than in some confounded treatment effect.

\section{GENERAL DISCUSSION}

When a CS and US are paired in a response-independent fashion characteristic of Pavlovian conditioning, evidence of associative learning is typically reflected in multiple behavioral changes in the presence of the CS. Thus, when a tone or light CS is paired with a paraorbital-shock US, as in the present experiments, one can observe a conditioned eyeblink and a conditioned potentiation of the startle response and can reasonably assume that these two measures are but among many other indices of conditioning that might have been employed. In this context, the results of Experiments 1 and 2 are significant in supporting those studies (e.g., Tait \& Saladin, 1986; Vandercar \& Schneiderman, 1967) that indicate that there are important systematic dissociations among distinguishable classes of these measures.

It is well known that the indexes of conditioning that are classified as reflecting a conditioned emotional response can be detected after fewer CS-US pairings and under conditions of longer CS-US training intervals than can measures of more specific reflexes such as the conditioned eyeblink (see, e.g., Lennertz \& Weinberger, 1992, for a review). These differences could be interpreted sim- 
ply as reflecting a greater sensitivity of the former, as compared with the latter, measures, and not necessarily as challenging their monotonic relationship. That the differences between the measures involve a theoretically more interesting divergence is perhaps most forcefully indicated by the findings of Tait and Saladin (1986), which have been replicated and extended in our laboratory (McNish, Betts, Brandon, \& Wagner, in press). They show that a backward conditioning protocol, where the CS follows the US, can produce a CER and a conditioned eyeblink tendency that are concurrently of opposite associative valence, with the former being excitatory when the latter is inhibitory. The present blocking data are likewise reflective of a dissociation between the two measures: When the location of the US was unchanged between pretraining and compound training in a customary blocking design, blocking was concurrently observed in the CER and the conditioned eyeblink measures; when the location of the US was shifted between the two phases, blocking was still potent in the CER but was abolished in the conditioned eyeblink.

One general way to account for this pattern of findings is through the assumptions made by Konorski (1967)that effective Pavlovian USs are represented by multiple nodes in the memory system, and that those that are identified with the emotional characteristics of the US (and assumed to be controlling of CERs) versus those that are identified with the remaining sensory and perceptual characteristics of the US (and assumed to be controlling of more specific CRs) become associated independently with correlated CSs. Konorski did not offer any mechanistic rationalization of why the two classes of nodes might show the different patterns of conditioning that they do. However, Wagner and Brandon (1989) showed how certain of the divergences might be theoretically accounted for in terms of an elaboration upon a stochastic model (SOP, Wagner, 1981) that assumes that the two classes of nodes differ in the parameters that govern their temporal activity dynamics (with the activity of emotional nodes being more persistent than that of sensory nodes). The present blocking data require no special assumptions beyond supposing that the shift in the location of the US between the pretraining and compound phases of Experiments 1 and 2 led to the activation of different sensory/perceptual nodes, but the same emotional node, representing the USs in the two phases. The pattern of results could then be accounted for by an extension of familiar reasoning regarding blocking, assuming that variation in associative learning is a result of variation in US processing, but occurring separately for the different US components. Blocking would be expected in a given response measure, consistent with the formulations of Rescorla and Wagner (1972) and Wagner (1978, 1981), to the degree that the memorial representation of the US component responsible for that response measure is associatively preactivated by the constellation of CSs.

Whereas the data of Experiments 1 and 2 can thus be viewed as consistent with a US-centered, variablereinforcement interpretation of blocking, they would seem to severely strain a CS-centered, attentional interpretation of the phenomenon. If one supposes that blocking occurs because the CS of interest is not processed during the compound phase of training, one would reasonably expect that blocking would be similarly evidenced in any response to the CS that is appropriate to measure the effects of compound training. In order to provide an attentional account of the present data from the shifted US condition, one would have to suppose that a CS can be unattended to for learning indexed by potentiated startle but be concurrently attended to for learning indexed by conditioned eyeblink. This would be unlike any attentional reasoning of which we are aware.

The various findings on the dissociation of response measures, including the data of Experiments 1 and 2, could encourage a more sweeping judgment concerning current theoretical preferences. Most theoretical discussion of blocking has occurred with the implicit assumption that the essential associations in Pavlovian conditioning are between representations of CSs and USs-that is, that they are "S-S" in character. A general outcome of such characterization has been an invitation to think of different indices of conditioning as alternative, correlated products of the same association. When different response measures turn out to have a substantial independence, one can suppose, as we have been led to do, that different ones reflect different $S-S$ associations, involving different, partially independent stimulus aspects. But under these circumstances one should also consider the alternative, that the different behavior measures reflect different $\mathrm{S}-\mathrm{R}$ associations. One might then be encouraged to anticipate that blocking would be seen differentially in different responses. Thus, in the present experiments, one might have expected blocking of the potentiated startle in either the standard or the shifted US condition, because the association of the pretrained cue with that response was well developed at the start of compound training. Likewise, one might have expected blocking of the eyeblink response in the standard US condition, because the eye that was conditioned during the compound phase was well conditioned at the start of compound training. However, one would not have expected blocking of conditioned eyeblink in the shifted US condition, because the eye that was reinforced during the compound phase was untrained at the start of compound training.

It is notable that Rescorla (1969) reasoned much like this (although neutrally as to the nature of the underlying associations) that blocking might be differentially observed in different ones of the multiple responses that can result from compound training. He pointed out, as an example, that the acquisition of different conditioned responses can occur at different rates so that, at the start of compound training in a conventional blocking sequence, one response might be relatively well developed when another was relatively undeveloped. In this case, Rescorla anticipated that the conditioned responses that are highly conditioned should be blocked in acquisition to the added CS, whereas those that are poorly conditioned should not be blocked. As a general rule for how 
much conditioning should be observed on a given response measure during compound conditioning, Rescorla recommended evaluating the discrepancy between the CR elicited at the start of compound conditioning and the maximum CR that could be supported by the US during such training-the greater the discrepancy, the more conditioning (i.e., the less blocking) should be expected. As an empirical generalization, this proposition would certainly cover the present results: Blocking occurred in potentiated startle, under both the standard and shifted US conditions, and in conditioned eyeblink, under the standard condition, where the discrepancy was presumably small; blocking did not occur in conditioned eyeblink under the shifted US condition, where the discrepancy was demonstrably large.

Donahoe, Crowly, Millard, and Stickney (1982) espoused a response-specific discrepancy rule for blocking similar to that suggested by Rescorla (1969), but recommended the essential discrepancy as being between the level of the CR occasioned by the constellation of CSs and the level of the UR produced by the US. The utility of this notion obviously depends on an identification of the UR. For example, the prominent, initial blink UR to paraorbital shock is much more bilateral than is the CR (see, e.g., McCormick, Lavond, \& Thompson, 1982), so that one might not thereby anticipate differential blocking in subjects pretrained on one eye versus the other.

Although some response-specific discrepancy rule may be more useful for summarizing the conditions under which blocking does or does not occur than an alternative that implies that all measures should covary, there are numerous cases in which the general view also seems lacking. Ganesan and Pearce (1988), for instance, observed blocking of magazine-approach behavior under a change in location of an appetitive US between the pretraining and compound phases, in spite of the fact that the different US locations led to clear differences in the topography of the approach responses in the two phases. Other challenging data come from conditioning circumstances in which the character of the CR varies dramatically with the quality of the CS-for example, between "head jerking" to a tone and "rearing" to a light, when either is paired with the same appetitive US. It has frequently been reported (e.g., by Blanchard \& Honig, 1976; Holland, 1977; Leyland \& Mackintosh, 1978; Tomie, 1981) that a pretrained CS can block the conditioning to an added CS, even though the pretrained CS does not evoke the same behavior that would normally be acquired by the added CS during compound training.

The challenge is to arrive at a description of the associative entities in Pavlovian conditioning such that the various antagonisms and facilitations that one observes under different conditions of training and testing can be anticipated. There is considerable evidence that conditioned emotional responses are acquired separately from specific conditioned reflexes such as the eyeblink and have a pervasive modulating influence on conditioned and unconditioned behaviors that would not be anticipated from specific reflex interactions. That blocking of the conditioned emotional response occurs, but blocking of the conditioned eyeblink does not, when there is a shift in the location of the reinforcing US between the pretraining and compound phases, is further witness to their associative separation. The outcomes are as one would expect if the CER reflects an association between the CS and the emotional aspects of the US, which does not change with a change in US location, whereas the eyeblink CR reflects an association between the CS and the remaining sensory/perceptual aspects of the US, which does change with a change in US location.

\section{REFERENCES}

Bakal, C. W., Johnson, R. D., \& Rescorla, R. A. (1974). The effect of change in US quality on the blocking effect. Pavlovian Journal, 9 , 97-103.

BlanchaRd, R., \& Honig, W. K. (1976). Surprise value of food determines its effectiveness as a reinforcer. Journal of Experimental Psychology: Animal Behavior Processes, 2, 67-74

Brandon, S. E., BetTs, S. L., \& Wagner, A. R. (1994). Discriminated, lateralized, eyeblink conditioning in the rabbit: An experimental context for separating specific and general associative influences. Joumal of Experimental Psychology: Animal Behavior Processes, 20, 292-307.

DiCKINSON, A. (1980). Contemporary animal learning theory. New York: Cambridge University Press.

Donahoe, J. W., Crowley, M. A., Millard, W. J., \& Stickney, K. A. (1982). A unified principle of reinforcement. In M. L. Commons, R. J. Herrnstein, \& H. Rachlin (Eds.), Quantitative analysis of behavior: Vol. 2. Matching and maximizing accounts (pp. 493-521). Cambridge, MA: Ballinger.

FALLS, W. A., \& WAGNER, A. R. (1991). Airpuff-elicited startle in the rabbit (Oryctologus cuniculus). Unpublished manuscript.

Ganesan, R., \& PeARCE, J. M. (1988). Effect of changing the unconditioned stimulus on appetitive blocking. Journal of Experimental $P$ sychology: Animal Behavior Processes, 14, 280-291.

Gormezano, I., \& GibBs, C. M. (1988). Transduction of the rabbit's nictitating membrane response. Behavior Research Methods, Instruments, \& Computers, 20, 18-21.

Holland, P. C. (1977). Conditioned stimulus as a determinant of the form of the Pavlovian conditioned response. Journal of Experimental Psychology: Animal Behavior Processes, 3, 77-104.

KAMIN, L. J. (1968). Attention-like processes in classical conditioning. In M. R. Jones (Ed.), Miami Symposium on the Prediction of Behavior: Aversive stimulation (pp. 9-32). Coral Gables, FL: University of Miami Press.

KoNORSKI, J. (1967). Integrative activity of the brain. Chicago: University of Chicago Press.

LASHLEY, K. S. (1942). An examination of the "continuity theory" as applied to discrimination learning. Journal of General Psychology, 26, $241-265$.

LenNertz, R. S., \& Weinberger, N. M. (1992). Analysis of response systems in Pavlovian conditioning reveals rapidly versus slowly acquired conditioned responses: Support for two factors, implications for behavior and neurobiology. Psychobiology, 20, 93-119.

LEYland, C. M., \& Mackintosh, N. J. (1978). Blocking of first-and second-order autoshaping in pigeons. Animal Learning \& Behavior, 6, 391-394.

Mackintosh, N. J. (1975). A theory of attention: Variations in the associability of stimuli with reinforcement. Psychological Review, 82, 276-298

Mackintosh, N. J. (1983). Conditioning and associative learning. New York: Oxford University Press.

McCormick, D. H., Lavond, D. G., \& Thompson, R. F. (1982). Concomitant classical conditioning of the rabbit nictitating membrane and eyelid responses: Correlation and implications. Physiology $\& B e-$ havior, 28, 769-775. 
McNish, K., Betts, S. L., Brandon, S. E., \& Wagner, A. R. (in press), Divergence of conditioned eyeblink and conditioned fear in backward Pavlovian training. Animal Learning \& Behavior.

PEARCE, J. M. (1987). An introduction to animal cognition. Hove, U.K.: Erlbaum.

Pearce, J. M., \& Hall, G. (1980). A model for Pavlovian learning: Variations in the effectiveness of conditioned but not of unconditioned stimuli. Psychological Review, 106, 532-552.

RESCORLA, R. A. (1969). Conditioned inhibition of fear. In W. K. Honig \& N. J. Mackintosh (Eds.), Fundamental issues in associative learning (pp. 65-89). Halifax, NS: Dalhousie University Press.

ResCorla, R. A., \& WAGNer, A. R. (1972). A theory of Pavlovian conditioning: Variations in the effectiveness of reinforcement and nonreinforcement. In A. H. Black \& W. F. Prokasy (Eds.), Classical conditioning II (pp, 64-99). New York: Appleton-Century-Crofts.

StickneY, K. J., \& DONAHOE, J. W. (1983). Attenuation of blocking by a change in US locus. Animal Learning \& Behavior, 11, 60-66.

Sutherland, N. S., \& Mackintosh, N. J. (1971). Mechanisms of animal discrimination learning. New York: Academic Press.

Tait, R. W., \& Saladin, M. E. (1986). Concurrent development of excitatory and inhibitory associations during backward conditioning. Animal Learning \& Behavior, 14, 133-137.

TOMIE, A. (1981). Effects of unpredictable food on the subsequent acquisition of autoshaping: Analysis of the context blocking hypothesis. In C. M. Locurto, H. S. Terrace, \& J. Gibbon (Eds.), Autoshaping and conditioning theory (pp. 181-215). New York: Academic Press.

Vandercar, D. H., \& SChneiderman, N. (1967). Interstimulus interval functions in different response systems during classical discrimination conditioning of rabbits. Psychonomic Science, 9, 9-10.

WAGNER, A. R. (1969). Stimulus-selection and a "modified continuity theory." In G. H. Bower \& J. T. Spence (Eds.), The psychology of learning and motivation (Vol. 3, pp. 1-40). New York: Academic Press.
WAGner, A. R. (1976). Priming in STM: An information-processing mechanism for self-generated or retrieval-generated depression in performance. In T. J. Tighe \& R. N. Leaton (Eds.), Habituation: Perspectives from child development, animal behavior, and neurophysiology (pp. 95-128). Hillsdale, NJ: Erlbaum.

WAGNER, A. R. (1978). Expectancies and the priming of STM. In S. H. Hulse, H. Fowler, \& W. K. Honig (Eds.), Cognitive aspects of animal behavior (pp. 177-209). Hillsdale, NJ: Erlbaum.

WAGNER, A. R. (1981). SOP: A model of automatic memory processing in animal behavior. In N. E. Spear \& R. R. Miller (Eds.), Information processing in animals: Memory mechanisms (pp. 5-47). Hillsdale, NJ: Erlbaum.

WAGNER, A. R., \& Brandon, S. E. (1989). Evolution of a structured connectionist model of Pavlovian conditioning (AESOP). In S. B. Klein \& R. R. Mowrer (Eds.), Contemporary learning theories: Pavlovian conditioning and the status of traditional learning theory (pp. 149-189). Hillsdale, NJ: Erlbaum.

WAGNeR, A. R., \& RESCORLA, R. A. (1972). Inhibition in Pavlovian conditioning: Application of a theory. In R. A. Boakes \& M. S. Haliday (Eds.), Inhibition and learning (pp. 301-336). New York: Academic Press.

\section{NOTE}

1. The side-to-side head shake response to a continuous stream of air is quite distinguishable from the up-and-down head movement component of the startle response to a brief pulse of air (Falls \& Wagner, 1991). Testing of the former simply allowed the experimenter to be confident of a clear air path directed to a sensitive surface.

(Manuscript received July 27, 1995; revision accepted for publication February 8,1996 .) 